\title{
Perturbed Skorohod equations and perturbed reflected diffusion processes
}

\author{
R.A. Doney *, T. Zhang \\ Department of Mathematics, University of Manchester, Oxford Road, Manchester M13 9PL, England, UK
}

Received 11 August 2003; received in revised form 11 March 2004; accepted 30 March 2004

Available online 11 September 2004

\begin{abstract}
In this paper, we obtain the existence and uniqueness of the solution to the perturbed Skorohod equation $g(t)=f(t)+$ $\alpha \max _{0 \leqslant s \leqslant t} g(s)+h(t)$ for any real constant $\alpha<1$ and any given continuous function $f$, where $g \geqslant 0$, and $h$ is an increasing function which only increases at the time $g$ is at zero. As an application, we establish the existence and uniqueness of some perturbed reflected diffusion processes.
\end{abstract}

(C) 2004 Elsevier SAS. All rights reserved.

\section{Résumé}

Dans ce papier, nous obtenons un résultat d'existence et d'unicité pour l'équation de Skorohod perturbée $g(t)=f(t)+$ $\alpha \max _{0 \leqslant s \leqslant t} g(s)+h(t)$ étant donnés un réel $\alpha<1$ et une fonction continue $f, g$ est une fonction positive et $h$ est une fonction croissante, strictement croissante aux points où $g$ s'annule. A titre d'appplication, nous obtenons l'existence et l'unicité de certains processus de diffusion réfléchis perturbés.

(c) 2004 Elsevier SAS. All rights reserved.

MSC: $60 \mathrm{H} 10 ; 60 \mathrm{~J} 60 ; 34 \mathrm{~A} 12 ; 34 \mathrm{~A} 40$

Keywords: Perturbed Skorohod equations; Local time; Reflection principle; Weak convergence; Reflected diffusions; Stratonovich integration

\section{Introduction}

There now exists a considerable body of literature devoted to the study of "perturbed" versions of familiar stochastic and deterministic equations; see e.g. [1-10,12-14]. An example is the perturbed Skorokhod equation

$$
X_{t}=x+B_{t}+\alpha \max _{0 \leqslant s \leqslant t} X_{s}+L_{t}^{X}, \quad t \geqslant 0,
$$

\footnotetext{
* Corresponding author.

E-mail address: rad@maths.man.ac.uk (R.A. Doney).
} 
where $B$ is a standard Brownian motion starting from $0, \alpha<1$ is a real constant, $x \geqslant 0$, and $L^{X}$ denotes a local time at zero of $X$. Since for $\alpha=0$ the solution is reflected Brownian motion any solution of (1) is referred to as a perturbed reflected Brownian motion, and the question of its existence and pathwise uniqueness has exercised several authors. As was pointed out in [10], if either $x>0$ or $\alpha<1 / 2$, an affirmative answer follows from the fact that the obvious deterministic version of (1) has a unique solution when $B$ is replaced by an arbitrary continuous function, vanishing at zero. The remaining case was settled in [3], a slightly weaker result being published simultaneously in [6]. However neither of these results dealt with the deterministic version with $x=0$ and $\alpha \in[1 / 2,1)$, and our first main result, Theorem 3.1 fills this gap, by means of completely different arguments.

Our other main result seems to be the first to deal with the analogous question for a general diffusion. Specifically we study the equation

$$
X_{t}=x+\int_{0}^{t} \sigma\left(X_{s}\right) d B_{s}+\alpha \max _{0 \leqslant s \leqslant t} X_{s}+L_{t}^{X}, \quad t \geqslant 0,
$$

where $\sigma$ is a Lipschitz continuous function on $R$. Again the case $x>0$ turns out to be relatively straight-forward, once we have established existence and pathwise uniqueness for perturbed diffusion processes in Theorem 2.1. For $x=0$ we exploit our result on the Skorohod equation together with Picard iteration to establish existence and pathwise uniqueness of a solution to (2), but unfortunately only in the case $\alpha<1 / 2$.

Finally we consider a version of (2) in which the Itô integral is replaced by a Stratonovich integral. In this case we are able to establish existence of a unique solution for all values of $\alpha<1$, under mild assumptions on $\sigma$.

\section{Perturbed diffusion processes}

Let $B_{t}, t \geqslant 0$ be a standard Brownian motion on a probability space $(\Omega, \mathcal{F}, P) . \mathcal{F}_{t}, t \geqslant 0$ denotes the filtration of the Brownian motion $B$. Let $\sigma(x), b(x)$ be Lipschitz continuous functions on $R$, i.e., there exists a constant $C$ such that

$$
\begin{aligned}
& |\sigma(x)-\sigma(y)| \leqslant C|x-y|, \\
& |b(x)-b(y)| \leqslant C|x-y| .
\end{aligned}
$$

For $\alpha<1$, consider the following stochastic differential equation:

$$
Y_{t}=Y_{0}+\int_{0}^{t} \sigma\left(Y_{s}\right) d B_{s}+\int_{0}^{t} b\left(Y_{s}\right) d s+\alpha \max _{0 \leqslant s \leqslant t} Y_{S} .
$$

Theorem 2.1. Assume that the random variable $Y_{0}$ is independent of $B$ and $E\left[\left|Y_{0}\right|^{2}\right]<\infty$. There exists a unique, continuous, $\mathcal{F}_{t}$-adapted solution $Y_{t}, t \geqslant 0$ to Eq. (5) such that $E\left[\sup _{0 \leqslant s \leqslant T}\left|Y_{s}\right|^{2}\right]<\infty$ for any $T>0$.

Proof. We construct the solution by iteration. Let

$$
Y_{t}^{0}=\frac{Y_{0}}{1-\alpha}, \quad 0 \leqslant t<\infty .
$$

For $n \geqslant 0$ define $Y_{t}^{n+1}$ to be the unique, continuous, adapted solution to the following equation:

$$
Y_{t}^{n+1}=Y_{0}+\int_{0}^{t} \sigma\left(Y_{s}^{n}\right) d B_{s}+\int_{0}^{t} b\left(Y_{s}^{n}\right) d s+\alpha \max _{0 \leqslant s \leqslant t} Y_{s}^{n+1} .
$$


Such a solution exists and can be expressed explicitly as

$$
Y_{t}^{n+1}=\frac{Y_{0}}{1-\alpha}+\int_{0}^{t} \sigma\left(Y_{s}^{n}\right) d B_{s}+\int_{0}^{t} b\left(Y_{s}^{n}\right) d s+\frac{\alpha}{1-\alpha} \max _{0 \leqslant s \leqslant t}\left(\int_{0}^{s} \sigma\left(Y_{u}^{n}\right) d B_{u}+\int_{0}^{s} b\left(Y_{u}^{n}\right) d u\right) .
$$

This is a consequence of the reflection principle. We will show that $Y^{n}$ converges uniformly on compact intervals almost surely. It follows from (7) that

$$
\begin{aligned}
\left|Y_{s}^{n+1}-Y_{s}^{n}\right| \leqslant & \left|\int_{0}^{s} \sigma\left(Y_{u}^{n}\right) d B_{u}-\int_{0}^{s} \sigma\left(Y_{u}^{n-1}\right) d B_{u}\right|+\left|\int_{0}^{s} b\left(Y_{u}^{n}\right) d u-\int_{0}^{s} b\left(Y_{u}^{n-1}\right) d u\right| \\
& +\frac{|\alpha|}{1-\alpha} \mid \max _{0 \leqslant v \leqslant s}\left(\int_{0}^{v} \sigma\left(Y_{u}^{n}\right) d B_{u}+\int_{0}^{v} b\left(Y_{u}^{n}\right) d u\right) \\
& -\max _{0 \leqslant v \leqslant s}\left(\int_{0}^{v} \sigma\left(Y_{u}^{n-1}\right) d B_{u}+\int_{0}^{v} b\left(Y_{u}^{n-1}\right) d u\right) \mid \\
\leqslant & \left|\int_{0}^{s} \sigma\left(Y_{u}^{n}\right) d B_{u}-\int_{0}^{s} \sigma\left(Y_{u}^{n-1}\right) d B_{u}\right|+\int_{0}^{s}\left|b\left(Y_{u}^{n}\right) d u-b\left(Y_{u}^{n-1}\right)\right| d u \\
& +\frac{|\alpha|}{1-\alpha} \max _{0 \leqslant v \leqslant s}\left|\int_{0}^{v}\left(\sigma\left(Y_{u}^{n}\right)-\sigma\left(Y_{u}^{n-1}\right)\right) d B_{u}\right| \\
& +\frac{|\alpha|}{1-\alpha} \max _{0 \leqslant v \leqslant s}\left|\int_{0}^{v}\left(b\left(Y_{u}^{n}\right)-b\left(Y_{u}^{n-1}\right)\right) d u\right|,
\end{aligned}
$$

where we used the fact that $\left|\max _{0 \leqslant v \leqslant s} f(v)-\max _{0 \leqslant v \leqslant s} g(v)\right| \leqslant \max _{0 \leqslant v \leqslant s}|f(v)-g(v)|$ holds for any two continuous functions $f$ and $g$. Thus,

$$
\max _{0 \leqslant s \leqslant t}\left|Y_{s}^{n+1}-Y_{s}^{n}\right| \leqslant\left(1+\frac{|\alpha|}{1-\alpha}\right)\left[\max _{0 \leqslant s \leqslant t}\left|\int_{0}^{s}\left(\sigma\left(Y_{u}^{n}\right)-\sigma\left(Y_{u}^{n-1}\right)\right) d B_{u}\right|+\int_{0}^{t}\left|b\left(Y_{u}^{n}\right)-b\left(Y_{u}^{n-1}\right)\right| d u\right] .
$$

In the rest of the proof, we will use $C$ to denote a generic constant which may change from line to line. By Burkhölder's inequality,

$$
\begin{aligned}
E\left[\max _{0 \leqslant s \leqslant t}\left|Y_{s}^{n+1}-Y_{s}^{n}\right|^{2}\right] & \leqslant C E\left[\int_{0}^{t}\left(\sigma\left(Y_{u}^{n}\right)-\sigma\left(Y_{u}^{n-1}\right)\right)^{2} d u\right]+C E\left[\int_{0}^{t}\left(b\left(Y_{u}^{n}\right)-b\left(Y_{u}^{n-1}\right)\right)^{2} d u\right] \\
& \leqslant C E\left[\int_{0}^{t}\left|Y_{u}^{n}-Y_{u}^{n-1}\right|^{2} d u\right] .
\end{aligned}
$$

Iterating the inequality we get that for fixed $T>0$,

$$
E\left[\max _{0 \leqslant s \leqslant T}\left|Y_{s}^{n+1}-Y_{s}^{n}\right|^{2}\right] \leqslant C \frac{T^{n}}{n !}
$$


since $E\left[Y_{0}^{2}\right]<\infty$. Consequently,

$$
P\left(\max _{0 \leqslant s \leqslant T}\left|Y_{s}^{n+1}-Y_{s}^{n}\right|>\frac{1}{2^{n}}\right) \leqslant C \frac{(4 T)^{n}}{n !} .
$$

By the Borel-Cantelli lemma, we see that $Y^{n}$ converges uniformly to a continuous, adapted process $Y$ on $[0, T]$ almost surely. Letting $n \rightarrow \infty$ in (6) it follows that $Y$ is a solution to Eq. (5). As $T$ is arbitrary, this proves the existence. Now suppose that $Y_{t}, t \geqslant 0$ and $Z_{t}, t \geqslant 0$ are two solutions to Eq. (5). Then we have, by the reflection principle,

$$
\begin{aligned}
& Y_{t}=\frac{Y_{0}}{1-\alpha}+\int_{0}^{t} \sigma\left(Y_{s}\right) d B_{s}+\frac{\alpha}{1-\alpha} \max _{0 \leqslant s \leqslant t}\left(\int_{0}^{s} \sigma\left(Y_{u}\right) d B_{u}+\int_{0}^{s} b\left(Y_{u}\right) d u\right), \\
& Z_{t}=\frac{Y_{0}}{1-\alpha}+\int_{0}^{t} \sigma\left(Z_{s}\right) d B_{s}+\frac{\alpha}{1-\alpha} \max _{0 \leqslant s \leqslant t}\left(\int_{0}^{s} \sigma\left(Z_{u}\right) d B_{u}+\int_{0}^{s} b\left(Z_{u}\right) d u\right) .
\end{aligned}
$$

Arguing as above, there is a constant $C$ such that

$$
\left|Y_{t}-Z_{t}\right| \leqslant C \max _{0 \leqslant s \leqslant t}\left|\int_{0}^{s}\left(\sigma\left(Y_{u}\right)-\sigma\left(Z_{u}\right)\right) d B_{u}\right|+C \int_{0}^{t}\left|b\left(Y_{u}\right)-b\left(Z_{u}\right)\right| d u .
$$

Applying Burkhölder's inequality,

$$
\begin{aligned}
E\left[\left|Y_{t}-Z_{t}\right|^{2}\right] & \leqslant C E\left[\int_{0}^{t}\left|\sigma\left(Y_{u}\right)-\sigma\left(Z_{u}\right)\right|^{2} d u\right]+C E\left[\int_{0}^{t}\left|b\left(Y_{u}\right)-b\left(Z_{u}\right)\right|^{2} d u\right] \\
& \leqslant C E\left[\int_{0}^{t}\left|Y_{u}-Z_{u}\right|^{2} d u\right]
\end{aligned}
$$

which implies $E\left[\left|Y_{t}-Z_{t}\right|^{2}\right]=0$ by Gronwall's inequality. Hence, the solution is unique.

\section{Perturbed Skorohod equation}

Set $W_{0}=\{f \in C([0, \infty) \rightarrow R) ; f(0)=0\}$ and $W^{+}=\{f \in C([0, \infty) \rightarrow R) ; f(t) \geqslant 0$ for all $t \geqslant 0\}$. The aim of this section is to prove

Theorem 3.1. Given $f \in W_{0}$ and $0 \leqslant \alpha<1$, there exist unique $g \in W^{+}$and $h \in W^{+}$such that

$$
g(t)=f(t)+\alpha \max _{0 \leqslant s \leqslant t} g(s)+h(t)
$$

(ii) $h(0)=0$ and $t \rightarrow h(t)$ is non-decreasing,

(iii) $\int_{0}^{t} \chi_{\{g(s)=0\}} d h(s)=h(t)$.

$(g, h)$ is called a solution to the perturbed Skorohod equation for the function $f$. 
Remark. This gives a simpler proof of Theorem 1 of [3].

For clarity, we split the proof of the theorem into several lemmas. For a continuous function $f$ and $0 \leqslant s \leqslant t$ define

$$
\omega_{s, t}(f)=\max _{s \leqslant u, v \leqslant t}(f(u)-f(v))=\max _{s \leqslant u, v \leqslant t}(|f(u)-f(v)|) .
$$

Lemma 3.2. Let $(g, h)$ be a solution to the perturbed Skorohod equation for the function $f$. There exists a constant $C$ independent of $g$ and $f$ such that

$$
\omega_{s, t}(g) \leqslant C \omega_{s, t}(f) \text { for all } 0 \leqslant s \leqslant t .
$$

Proof. Fix $0 \leqslant s<t$. We observe that if $g(v)>0$ for all $v \in(s, t)$ then

$$
g(v)-g(s)=f(v)-f(s)+\alpha\left(\max _{0 \leqslant u \leqslant v} g(u)-\max _{0 \leqslant u \leqslant s} g(u)\right) \quad \text { for all } v \in[s, t],
$$

and if $\max _{0 \leqslant u \leqslant t} g(u)=\max _{0 \leqslant u \leqslant s} g(u)$

$$
g(v)-g(s)=f(v)-f(s)+h(v)-h(s) \quad \text { for all } v \in[s, t] .
$$

Without loss of generality we assume $\omega_{s, t}(g)>0$ and $\omega_{s, t}(g)=g\left(v_{0}\right)-g\left(u_{0}\right)$ for some $u_{0}, v_{0} \in[s, t]$.

Case 1. $u_{0}<v_{0}$.

Let $l_{0}=v_{0} \wedge \inf \left\{v \geqslant u_{0} ; g(v)=\max _{0 \leqslant u \leqslant v} g(u)\right\}$. As $\max _{0 \leqslant u \leqslant l_{0}} g(u)=\max _{0 \leqslant u \leqslant u_{0}} g(u)$, by (22),

$$
g(v)-g\left(u_{0}\right)=f(v)-f\left(u_{0}\right)+h(v)-h\left(u_{0}\right) \text { for all } v \in\left[u_{0}, l_{0}\right] .
$$

It follows by the reflection principle that for $v \in\left[u_{0}, l_{0}\right]$,

$$
\begin{aligned}
g(v)-g\left(u_{0}\right) & =f(v)-f\left(u_{0}\right)-\inf _{u_{0} \leqslant u \leqslant v}\left\{\left(g\left(u_{0}\right)+f(u)-f\left(u_{0}\right)\right) \wedge 0\right\} \\
& \leqslant f(v)-f\left(u_{0}\right)-\inf _{u_{0} \leqslant u \leqslant v}\left\{\left(f(u)-f\left(u_{0}\right)\right) \wedge 0\right\} \\
& \leqslant\left|f(v)-f\left(u_{0}\right)\right|+\max _{u_{0} \leqslant u \leqslant v}\left\{\left|f(u)-f\left(u_{0}\right)\right|\right\} \\
& \leqslant 2 \max _{u_{0} \leqslant u \leqslant v}\left\{\left|f(u)-f\left(u_{0}\right)\right|\right\} .
\end{aligned}
$$

In particular,

$$
g\left(l_{0}\right)-g\left(u_{0}\right) \leqslant 2 \max _{u_{0} \leqslant u \leqslant l_{0}}\left\{\left|f(u)-f\left(u_{0}\right)\right|\right\} .
$$

On the other hand, we can assume $g(u)>0$ for all $u \in\left[l_{0}, v_{0}\right]$. Otherwise, replace $u_{0}$ by $\bar{u}_{0}=\sup \{u ; u \leqslant$ $\left.v_{0}, g(u)=0\right\}$. Thus, by $(21)$

$$
g\left(v_{0}\right)-g\left(l_{0}\right)=f\left(v_{0}\right)-f\left(l_{0}\right)+\alpha\left(\max _{0 \leqslant u \leqslant v_{0}} g(u)-\max _{0 \leqslant u \leqslant l_{0}} g(u)\right) .
$$

If $l_{0}=v_{0}$, then (20) follows already from (25). Assume now $l_{0}<v_{0}$. In this case it is easy to see that $g\left(l_{0}\right)=$ $\max _{0 \leqslant u \leqslant l_{0}} g(u)$ and $g\left(v_{0}\right)=\max _{s \leqslant u \leqslant t} g(u)$. Therefore, we also have $g\left(v_{0}\right)=\max _{0 \leqslant u \leqslant v_{0}} g(u)$. Consequently,

$$
g\left(v_{0}\right)-g\left(l_{0}\right)=f\left(v_{0}\right)-f\left(l_{0}\right)+\alpha\left(g\left(v_{0}\right)-g\left(l_{0}\right)\right) .
$$

Hence,

$$
g\left(v_{0}\right)-g\left(l_{0}\right)=\frac{1}{1-\alpha}\left(f\left(v_{0}\right)-f\left(l_{0}\right)\right) .
$$

Combining (25) and (28) yields 


$$
\begin{aligned}
g\left(v_{0}\right)-g\left(u_{0}\right) & =g\left(v_{0}\right)-g\left(l_{0}\right)+g\left(l_{0}\right)-g\left(u_{0}\right) \\
& \leqslant \frac{1}{1-\alpha}\left(f\left(v_{0}\right)-f\left(l_{0}\right)\right)+2 \max _{u_{0} \leqslant u \leqslant l_{0}}\left\{\left|f(u)-f\left(u_{0}\right)\right|\right\} \\
& \leqslant\left(2+\frac{1}{1-\alpha}\right) \max _{s \leqslant u, v \leqslant t}\{|f(u)-f(v)|\} .
\end{aligned}
$$

Case 2. $v_{0}<u_{0}$.

We can assume $g(u)>0$ for all $u \in\left[v_{0}, u_{0}\right]$. Otherwise, we can replace $u_{0}$ by $\bar{u}_{0}=\inf \left\{u>v_{0} ; g(u)=0\right\}$. Thus, by (21),

$$
g\left(u_{0}\right)-g\left(v_{0}\right)=f\left(u_{0}\right)-f\left(v_{0}\right)+\alpha\left(\max _{0 \leqslant u \leqslant u_{0}} g(u)-\max _{0 \leqslant u \leqslant v_{0}} g(u)\right) .
$$

Since $g\left(v_{0}\right)=\max _{v_{0} \leqslant u \leqslant u_{0}} g(u)$, we have

$$
g\left(u_{0}\right)-g\left(v_{0}\right)=f\left(u_{0}\right)-f\left(v_{0}\right) \leqslant \omega_{s, t}(f) .
$$

Combining case 1 and case 2 completes the proof.

Lemma 3.3. Let $\left(g_{n}, h_{n}\right)$ be a solution to the perturbed Skorohod equation for a function $f_{n}$. If $f_{n} \rightarrow f$ uniformly on compact intervals as $n \rightarrow \infty$, then a solution $(g, h)$ to the perturbed Skorohod equation for the function $f$ exists.

Proof. Without loss of generality, we can restrict ourself to the finite interval $[0,1]$. Since $f_{n} \rightarrow f$ as $n \rightarrow \infty$, $\left\{f_{n}, n \geqslant 1\right\}$ is a bounded set of equicontinuous functions. By Lemma 3.2, we see that $K=\left\{g_{n}, n \geqslant 1\right\}$ is also a bounded set of equicontinuous functions. By the Arzela-Ascoli theorem, there exists a subsequence $g_{n_{k}}, k \geqslant 1$, that converges uniformly to a continuous function $g$ on [0,1]. It is seen from (18) that $h_{n_{k}}, k \geqslant 1$ also converges uniformly to a non-decreasing continuous function $h$ on $[0,1]$ and

$$
g(t)=f(t)+\alpha \max _{0 \leqslant s \leqslant t} g(s)+h(t) .
$$

To show that $(g, h)$ is a solution to the perturbed Skorohod equation for the function $f$, it remains to prove that $\int_{0}^{t} \chi\{g(s)=0\} d h(s)=h(t)$ for $t \geqslant 0$. To see this it is enough to show that for any continuous function $l(t)$ on $(0, \infty)$ with compact support, $\int_{0}^{t} l(g(s)) d h(s)=0$. This in fact follows from

$$
\int_{0}^{t} l(g(s)) d h(s)=\lim _{k \rightarrow \infty} \int_{0}^{t} l\left(g_{n_{k}}(s)\right) d h_{n_{k}}(s)=0 .
$$

\section{Proof of Theorem 3.1.}

Existence. Again we restrict ourself to the interval $[0,1]$. For the existence, by Lemma 3.3 we can assume $f \in H_{2}^{1}[0,1]$, where

$$
H_{2}^{1}[0,1]:=\left\{f ; f:[0,1] \rightarrow R \text { is absolutely continuous and } \int_{0}^{1} f^{\prime}(s)^{2} d s<\infty\right\},
$$

where $f^{\prime}(s)$ stands for the derivative of $f$. We will use the so called penalized method. Define

$$
\phi(x)= \begin{cases}0 & \text { if } x \geqslant 0 \\ x^{2} & \text { if } x<0\end{cases}
$$


Consider the following functional equation

$$
g_{\varepsilon}(t)=f(t)+\alpha \max _{0 \leqslant s \leqslant t} g_{\varepsilon}(s)-\frac{1}{\varepsilon} \int_{0}^{t} \phi^{\prime}\left(g_{\varepsilon}(s)\right) d s,
$$

where $\phi^{\prime}(x)$ stands for the derivative of $\phi$.

Note that there is no "local time" term in Eq. (35). The existence of the solution of Eq. (35) can be obtained in exactly the same way as Theorem 2.1. In fact, let

$$
g_{\varepsilon}^{0}(t)=0, \quad 0 \leqslant t<\infty,
$$

and define $g_{\varepsilon}^{n+1}(t)$ to be the unique continuous solution of the equation:

$$
g_{\varepsilon}^{n+1}(t)=f(t)-\frac{1}{\varepsilon} \int_{0}^{t} \phi^{\prime}\left(g_{\varepsilon}^{n}(s)\right) d s+\alpha \max _{0 \leqslant s \leqslant t} g_{\varepsilon}^{n+1}(s) .
$$

By the reflection principle,

$$
g_{\varepsilon}^{n+1}(t)=f(t)-\frac{1}{\varepsilon} \int_{0}^{t} \phi^{\prime}\left(g_{\varepsilon}^{n}(s)\right) d s+\frac{\alpha}{1-\alpha} \max _{0 \leqslant s \leqslant t}\left(f(s)-\frac{1}{\varepsilon} \int_{0}^{s} \phi^{\prime}\left(g_{\varepsilon}^{n}(u)\right) d u\right) .
$$

Following the proof of Theorem 2.1 sentence by sentence, we arrive at

$$
\begin{aligned}
\max _{0 \leqslant s \leqslant t}\left|g_{\varepsilon}^{n+1}(t)-g_{\varepsilon}^{n}(t)\right| & \leqslant\left(1+\frac{|\alpha|}{1-\alpha}\right) \frac{1}{\varepsilon} \int_{0}^{t}\left|\phi^{\prime}\left(g_{\varepsilon}^{n}(s)\right)-\phi^{\prime}\left(g_{\varepsilon}^{n-1}(s)\right)\right| d s \\
& \leqslant\left(1+\frac{|\alpha|}{1-\alpha}\right) C_{\varepsilon} \int_{0}^{t}\left|g_{\varepsilon}^{n}(s)-g_{\varepsilon}^{n-1}(s)\right| d s .
\end{aligned}
$$

Iterating the above inequality as in the proof of Theorem 2.1, it is seen that $g_{\varepsilon}^{n}$ converges uniformly on any finite interval $[0, T]$ to a continuous function $g_{\varepsilon}$, which clearly is a solution of Eq. (35).

Put $A^{\varepsilon}(t)=\max _{0 \leqslant s \leqslant t} g_{\varepsilon}(s)$, then

$$
\phi\left(g_{\varepsilon}(t)\right)+\frac{1}{\varepsilon} \int_{0}^{t} \phi^{\prime}\left(g_{\varepsilon}(s)\right)^{2} d s=\int_{0}^{t} \phi^{\prime}\left(g_{\varepsilon}(s)\right) f^{\prime}(s) d s+\alpha \int_{0}^{t} \phi^{\prime}\left(g_{\varepsilon}(s)\right) d A^{\varepsilon}(s) .
$$

Since $\phi^{\prime}(x) \leqslant 0$ and $A^{\varepsilon}(t)$ is increasing in $t$, it follows from (36) that

$$
\frac{1}{\varepsilon} \int_{0}^{t} \phi^{\prime}\left(g_{\varepsilon}(s)\right)^{2} d s \leqslant \int_{0}^{t} \phi^{\prime}\left(g_{\varepsilon}(s)\right) f^{\prime}(s) d s \leqslant\left(\int_{0}^{t} \phi^{\prime}\left(g_{\varepsilon}(s)\right)^{2} d s\right)^{\frac{1}{2}}\left(\int_{0}^{t} f^{\prime}(s)^{2} d s\right)^{\frac{1}{2}} .
$$

Consequently,

$$
\frac{1}{\varepsilon^{2}} \int_{0}^{t} \phi^{\prime}\left(g_{\varepsilon}(s)\right)^{2} d s \leqslant \int_{0}^{t} f^{\prime}(s)^{2} d s
$$

and furthermore, by the Hölder inequality and (38), 


$$
\begin{aligned}
\phi\left(g_{\varepsilon}(t)\right) & \leqslant \int_{0}^{t} \phi^{\prime}\left(g_{\varepsilon}(s)\right) f^{\prime}(s) d s \\
& \leqslant\left(\int_{0}^{t} \phi^{\prime}\left(g_{\varepsilon}(s)\right)^{2} d s\right)^{\frac{1}{2}}\left(\int_{0}^{t} f^{\prime}(s)^{2} d s\right)^{\frac{1}{2}} \leqslant \varepsilon \int_{0}^{t} f^{\prime}(s)^{2} d s .
\end{aligned}
$$

Set $h^{\varepsilon}(t)=-\frac{1}{\varepsilon} \int_{0}^{t} \phi^{\prime}\left(g_{\varepsilon}(s)\right) d s$. Then by (38) there exists a sequence $\varepsilon_{n} \rightarrow 0$ such that $h^{\varepsilon_{n}}(t)$ converges uniformly to $h(t) \in H_{2}^{1}[0,1]$. On the other hand, it follows from Eq. (35) that

$$
\begin{aligned}
\left|g_{\varepsilon_{n}}(t)-g_{\varepsilon_{m}}(t)\right| & \leqslant \alpha\left|A^{\varepsilon_{n}}(t)-A^{\varepsilon_{m}}(t)\right|+\left|h^{\varepsilon_{n}}(t)-h^{\varepsilon_{m}}(t)\right| \\
& \leqslant \alpha \max _{0 \leqslant s \leqslant t}\left|g_{\varepsilon_{n}}(s)-g_{\varepsilon_{m}}(s)\right|+\left|h^{\varepsilon_{n}}(t)-h^{\varepsilon_{m}}(t)\right| .
\end{aligned}
$$

Hence,

$$
\max _{0 \leqslant s \leqslant 1}\left|g_{\varepsilon_{n}}(s)-g_{\varepsilon_{m}}(s)\right| \leqslant \frac{1}{1-\alpha} \max _{0 \leqslant s \leqslant 1}\left|h_{\varepsilon_{n}}(s)-h_{\varepsilon_{m}}(s)\right| .
$$

Therefore, $\left\{g_{\varepsilon_{m}}(t), m \geqslant 1\right\}$ is a Cauchy sequence in $C([0,1])$. Let $g$ denote the limit. We will show that $(g, h)$ is a solution to the perturbed Skorohod equation for the function $f$. First of all, it is clear from the construction that

$$
g(t)=f(t)+\alpha \max _{0 \leqslant s \leqslant t} g(s)+h(t),
$$

and $h(t)$ is a non-negative increasing function (because $h_{\varepsilon_{n}}$ is). Secondly, it follows from (39) that $\phi(g(t))=0$, which implies that $g(t) \geqslant 0$ for all $t \geqslant 0$. Now for any continuous function $l(t)$ on $(0, \infty)$ with compact support, say that the support is contained in $\left[\delta_{1}, \delta_{2}\right]$ for some $\delta_{1}>0$, we have

$$
\begin{aligned}
\left|\int_{0}^{t} l\left(g_{\varepsilon_{n}}(s)\right) d h^{\varepsilon_{n}}(s)\right| & \leqslant \int_{0}^{t}\left|l\left(g_{\varepsilon_{n}}(s)\right)\right| d h^{\varepsilon_{n}}(s)=\frac{1}{\varepsilon_{n}} \int_{0}^{t}\left|l\left(g_{\varepsilon_{n}}(s)\right)\right|\left|\phi^{\prime}\left(g_{\varepsilon_{n}}(s)\right)\right| d s \\
& \leqslant C \int_{0}^{t} \chi_{\left[\delta_{1}, \delta_{2}\right]}\left(g_{\varepsilon_{n}}(s)\right)\left|\phi^{\prime}\left(g_{\varepsilon_{n}}(s)\right)\right| d s=0,
\end{aligned}
$$

since $\phi^{\prime}(x)=0$ for $x>0$.

The identity $\int_{0}^{t} \chi\{g(s)=0\} d h(s)=h(t)$ for $t \geqslant 0$ can now be proved similarly as in the proof of Lemma 3.3. Thus, we complete the proof of existence.

Uniqueness: For any $\delta>0$, let $g_{\delta}(t)$ be the unique solution to equation

$$
g_{\delta}(t)=(1-\alpha) \delta+f(t)+\alpha \max _{0 \leqslant s \leqslant t} g_{\delta}(s)+h_{\delta}(t) .
$$

Such an equation was studied in [10] and [3]. Let $(g, h)$ be a solution to Eq. (18). A "round trip" of $g$ is defined to be a section of the path $g$ lying between two maxima and containing a visit to zero. It follows from the proof of Lemma 3 in [3] that

$$
\sup _{0 \leqslant s \leqslant t}\left|g_{\delta}(s)-g(s)\right| \leqslant \delta\left(\alpha^{*}+1\right)^{2 n_{t}+1},
$$

where $\alpha^{*}=\frac{\alpha}{1-\alpha}, n_{t}$ is the number of round trips completed by $g$ by time $t$. This implies that $g(t)=\lim _{\delta \rightarrow 0} g_{\delta}(t)$, which gives the uniqueness. 


\section{Perturbed reflected diffusions}

Let $\sigma$ be as in Section 2. For $x \geqslant 0$, consider the stochastic differential equation:

$$
X_{t}=x+\int_{0}^{t} \sigma\left(X_{s}\right) d B_{s}+\alpha \max _{0 \leqslant s \leqslant t} X_{s}+L_{t} .
$$

Definition 4.1. We say that $\left(X_{t}, L_{t}, t \geqslant 0\right)$ is a solution to Eq. (45) if

(i) $X_{0}=x, X_{t} \geqslant 0$ for $t \geqslant 0$,

(ii) $X_{t}, L_{t}$ are continuous and adapted to the filtration of $B$,

(iii) $L_{t}$ is non-decreasing with $L_{0}=0$ and

$$
\int_{0}^{t} \chi_{\left\{X_{s}=0\right\}} d L_{s}=L_{t},
$$

(iv) $\left(X_{t}, L_{t}, t \geqslant 0\right)$ satisfies Eq. (45) almost surely for every $t>0$.

The cases $x=0$ and $x>0$ are quite different. We will treat them separately.

Theorem 4.2. Assume $\alpha<1$ and $\sigma$ is Lipschitz. If $x>0$, there exists a unique solution $\left(X_{t}, L_{t}, t \geqslant 0\right)$ to Eq. (45).

Proof. We construct the solution iteratively in a similar way to [10]. Define $Y_{t}^{0}$ to be the unique solution to the equation:

$$
Y_{t}^{0}=x+\int_{0}^{t} \sigma\left(Y_{s}^{0}\right) d B_{s}+\alpha \max _{0 \leqslant s \leqslant t} Y_{s}^{0} .
$$

It is known from Section 2 that such a solution exists. Set $T_{1}=\inf \left\{t \geqslant 0 ; Y_{t}^{0}=0\right\}$. Then $T_{1}>0$ a.s. as $x>0$. Define

$$
X_{t}=Y_{t}^{0}, \quad L_{t}=0 \quad \text { for } 0 \leqslant t \leqslant T_{1} .
$$

Put $B_{t}^{1}=B_{t+T_{1}}-B_{T_{1}}$ for $t \geqslant 0$. It is well known that $B_{t}^{1}, t \geqslant 0$ is a standard Brownian motion independent of $\mathcal{F}_{T_{1}}$. Consider the stochastic differential equation with reflecting boundary:

$$
\begin{aligned}
& Z_{t}^{1}=\int_{0}^{t} \sigma\left(Z_{s}^{1}\right) d B_{s}^{1}+L_{t}^{1}, \\
& Z_{t}^{1} \geqslant 0, \quad Z_{0}^{1}=0, \\
& L_{0}^{1}=0, \quad \int_{0}^{t} \chi_{\left\{Z_{s}^{1}=0\right\}} d L_{s}^{1}=L_{t}^{1} .
\end{aligned}
$$

The definition of a solution to this equation is the same as Definition 4.1 with $x=0$ and $\alpha=0$. It is known that a unique solution $\left(Z_{t}^{1}, L_{t}^{1}\right)$ to the Eq. (48) exists, see e.g. [11] or [15]. In general, suppose that $\left(X_{t}, L_{t}\right)$ has been defined for $0 \leqslant t \leqslant T_{2 n-1}$. We can construct $\left(X_{t}, L_{t}\right)$ for $T_{2 n-1} \leqslant t \leqslant T_{2 n+1}$ as follows. Let $Z_{t}^{2 n-1}$ be the solution to the equation: 


$$
\begin{aligned}
& Z_{t}^{2 n-1}=\int_{0}^{t} \sigma\left(Z_{s}^{2 n-1}\right) d B_{s}^{2 n-1}+L_{t}^{2 n-1}, \\
& Z_{t}^{2 n-1} \geqslant 0, \quad Z_{0}^{2 n-1}=0 \\
& L_{0}^{2 n-1}=0, \quad \int_{0}^{t} \chi_{\left\{Z_{s}^{2 n-1}=0\right\}} d L_{s}^{2 n-1}=L_{t}^{2 n-1},
\end{aligned}
$$

where $B_{t}^{2 n-1}=B_{t+T_{2 n-1}}-B_{T_{2 n-1}}$. Put $T_{2 n}=\inf \left\{t>T_{2 n-1} ; Z_{t-T_{2 n-1}}^{2 n-1}=\max _{0 \leqslant s \leqslant T_{2 n-1}} X_{s}\right\}$ and set

$$
X_{t}=Z_{t-T_{2 n-1}}^{2 n-1}, \quad L_{t}=L_{T_{2 n-1}}+L_{t-T_{2 n-1}}^{2 n-1} \text { for } T_{2 n-1} \leqslant t \leqslant T_{2 n} .
$$

Let $Y_{t}^{2 n}$ denote the solution to equation:

$$
Y_{t}^{2 n}=(1-\alpha) X_{T_{2 n}}+\int_{0}^{t} \sigma\left(Y_{s}^{2 n}\right) d B_{s}^{2 n}+\alpha \max _{0 \leqslant s \leqslant t} Y_{s}^{2 n},
$$

where $B_{t}^{2 n}=B_{t+T_{2 n}}-B_{T_{2 n}}$. Set $T_{2 n+1}=\inf \left\{t>T_{2 n}, Y_{t-T_{2 n}}=0\right\}$ and

$$
X_{t}=Y_{t-T_{2 n}}^{2 n}, \quad L_{t}=L_{T_{2 n}} \text { for } T_{2 n} \leqslant t \leqslant T_{2 n+1} .
$$

By this procedure, we obtain a sequence of increasing stopping times $T_{n}, n \geqslant 0$. Set $T=\lim _{n \rightarrow \infty} T_{n}$. Then $T$ is again a stopping time, and $\left(X_{t}, L_{t}\right)$ is a well defined continuous process for all $0 \leqslant t<T$. We will show that $\left(X_{t}, L_{t}, t<T\right)$ satisfies Eq. (45) in the sense of Definition 4.1. To achieve this, it is sufficient to prove that $\left(X_{t}, L_{t}\right)$ satisfies Eq. (45) for $T_{2 n} \leqslant t \leqslant T_{2 n+1}$ and $n=0,1 \ldots$ We will do this by induction. It is obvious that $\left(X_{t}, L_{t}\right)$ is a solution to Eq. (45) for $0 \leqslant t \leqslant T_{1}$. If $T_{1} \leqslant t \leqslant T_{2}$, it follows that

$$
\begin{aligned}
X_{t} & =Z_{t-T_{1}}^{1} \\
& =\int_{0}^{t-T_{1}} \sigma\left(Z_{s}^{1}\right) d B_{s}^{1}+L_{t-T_{1}}^{1} \\
& =\int_{0}^{t-T_{1}} \sigma\left(Z_{s}^{1}\right) d B_{s+T_{1}}+L_{t} \\
& =\int_{T_{1}}^{t} \sigma\left(X_{u}\right) d B_{u}+L_{t} \\
& =X_{T_{1}}+\int_{T_{1}}^{t} \sigma\left(X_{u}\right) d B_{u}+L_{t} \\
& =x+\int_{0}^{T_{1}} \sigma\left(X_{s}\right) d B_{s}+\alpha \max _{0 \leqslant s \leqslant T_{1}} X_{s}+\int_{T_{1}}^{t} \sigma\left(X_{u}\right) d B_{u}+L_{t} \\
& =x+\int_{0}^{t} \sigma\left(X_{s}\right) d B_{s}+\alpha \max _{0 \leqslant s \leqslant t} X_{s}+L_{t},
\end{aligned}
$$


since $\max _{0 \leqslant s \leqslant T_{1}} X_{s}=\max _{0 \leqslant s \leqslant t} X_{s}$ for $T_{1} \leqslant t \leqslant T_{2}$, and $X_{T_{1}}=0$.

Furthermore if $T_{1} \leqslant t \leqslant T_{2}$, we see that

$$
\int_{0}^{t} \chi_{\left\{X_{s}=0\right\}} d L_{s}=\int_{T_{1}}^{t} \chi_{\left\{X_{s}=0\right\}} d L_{s-T_{1}}^{1}=\int_{0}^{t-T_{1}} \chi_{\left\{Z_{s}^{1}=0\right\}} d L_{s}^{1}=L_{t-T_{1}}^{1}=L_{t} .
$$

Thus we have showed that $\left(X_{t}, L_{t}\right)$ is a solution to Eq. (45) for $0 \leqslant t \leqslant T_{2}$. Suppose that $\left(X_{t}, L_{t}\right)$ satisfies Eq. (45) for $0 \leqslant t \leqslant T_{2 n}$. If $T_{2 n} \leqslant t \leqslant T_{2 n+1}$, it follows that

$$
\begin{aligned}
X_{t} & =Y_{t-T_{2 n}}^{2 n} \\
& =(1-\alpha) X_{T_{2 n}}+\int_{0}^{t-T_{2 n}} \sigma\left(Y_{s}^{2 n}\right) d B_{s}^{2 n}+\alpha \max _{0 \leqslant s \leqslant t-T_{2 n}} Y_{s}^{2 n} \\
& =x+\int_{0}^{T_{2 n}} \sigma\left(X_{s}\right) d B_{s}+\alpha \max _{0 \leqslant s \leqslant T_{2 n}} X_{s}+L_{T_{2 n}}-\alpha X_{T_{2 n}}+\int_{0}^{t-T_{2 n}} \sigma\left(Y_{s}^{2 n}\right) d B_{s+T_{2 n}}+\alpha \max _{0 \leqslant s \leqslant t-T_{2 n}} Y_{s}^{2 n} \\
& =x+\int_{0}^{t} \sigma\left(X_{S}\right) d B_{s}+\alpha \max _{T_{2 n} \leqslant s \leqslant t} X_{s}+L_{t} \\
& =x+\int_{0}^{t} \sigma\left(X_{s}\right) d B_{s}+\alpha \max _{0 \leqslant s \leqslant t} X_{s}+L_{t},
\end{aligned}
$$

where we have used the fact that $X_{T_{2 n}}=\max _{0 \leqslant s \leqslant T_{2 n}} X_{s}$ and $Y_{0}^{2 n}=X_{T_{2 n}}$ from their definitions. Since $X_{t} \neq 0$ for $T_{2 n} \leqslant t<T_{2 n+1}$, we also have

$$
\int_{0}^{t} \chi_{\left\{X_{s}=0\right\}} d L_{s}=\int_{0}^{T_{2 n}} \chi_{\left\{X_{s}=0\right\}} d L_{s}=L_{T_{2 n}}=L_{t} .
$$

So $\left(X_{t}, L_{t}\right)$ satisfies Eq. (45) also for $T_{2 n} \leqslant t \leqslant T_{2 n+1}$. Repeating similar arguments as for (53), we also can show that $\left(X_{t}, L_{t}\right)$ satisfies Eq. (45) for $T_{2 n+1} \leqslant t \leqslant T_{2 n+2}$.

Finally we show that $T=\infty$ a.s. By the construction of $X$, we have that

$$
0=X_{T_{2 n+1}}=\max _{0 \leqslant s \leqslant T_{2 n}} X_{s}+\int_{T_{2 n}}^{T_{2 n+1}} \sigma\left(X_{s}\right) d B_{s}+\alpha\left(\max _{0 \leqslant s \leqslant T_{2 n+1}} X_{s}-\max _{0 \leqslant s \leqslant T_{2 n}} X_{S}\right)+L_{T_{2 n+1}}-L_{T_{2 n}} .
$$

Suppose $T<\infty$ with positive probability. Letting $n \rightarrow \infty$ in (56), we get $0=\max _{0 \leqslant s \leqslant T} X_{s}$ which contradicts the fact that $X_{0}=(1-\alpha)^{-1} x>0$. The proof of existence is complete.

On the other hand, it is easily seen that the solution is unique since it is unique on each interval $\left[T_{n}, T_{n+1}\right]$.

Theorem 4.3. Assume $x=0$. If $0 \leqslant \alpha<\frac{1}{2}$, then there exists a unique solution $\left(X_{t}, L_{t}, t \geqslant 0\right)$ to Eq. (45). 
Proof. We will use the Picard iteration method. Define $X_{t}^{0} \equiv 0$ and $\left(X_{t}^{n+1}, L_{t}^{n+1}\right)$ to be the unique solution to the equation:

$$
X_{t}^{n+1}=\int_{0}^{t} \sigma\left(X_{s}^{n}\right) d B_{s}+\alpha \max _{0 \leqslant s \leqslant t} X_{s}^{n+1}+L_{t}^{n+1} .
$$

The existence and uniqueness of this solution follow from Section 3. Observe that by the reflection principle,

$$
L_{t}^{n+1}=-\inf _{s \leqslant t}\left\{\left(\int_{0}^{s} \sigma\left(X_{u}^{n}\right) d B_{u}+\alpha \max _{0 \leqslant u \leqslant s} X_{u}^{n+1}\right) \wedge 0\right\} .
$$

Now (57) and (58) imply that

$$
\begin{aligned}
\left|X_{t}^{n+1}-X_{t}^{n}\right| \leqslant & \left|\int_{0}^{t}\left(\sigma\left(X_{s}^{n}\right)-\sigma\left(X_{s}^{n-1}\right)\right) d B_{s}\right|+\sup _{s \leqslant t}\left|\int_{0}^{s}\left(\sigma\left(X_{u}^{n}\right)-\sigma\left(X_{u}^{n-1}\right)\right) d B_{u}\right| \\
& +2 \alpha \sup _{s \leqslant t}\left|X_{s}^{n+1}-X_{s}^{n}\right| .
\end{aligned}
$$

Consequently,

$$
\sup _{s \leqslant t}\left|X_{s}^{n+1}-X_{s}^{n}\right| \leqslant \frac{2}{1-2 \alpha} \sup _{s \leqslant t}\left|\int_{0}^{s}\left(\sigma\left(X_{u}^{n}\right)-\sigma\left(X_{u}^{n-1}\right)\right) d B_{u}\right| .
$$

By Burkhölder's inequality,

$$
E\left[\sup _{s \leqslant t}\left|X_{s}^{n+1}-X_{s}^{n}\right|^{2}\right] \leqslant C_{\alpha} E\left[\int_{0}^{t}\left(\sigma\left(X_{s}^{n}\right)-\sigma\left(X_{s}^{n-1}\right)\right)^{2} d u\right] \leqslant C_{\alpha} E\left[\int_{0}^{t}\left(X_{s}^{n}-X_{s}^{n-1}\right)^{2} d u\right] .
$$

Thus, we deduce that for any fixed $T>0$,

$$
E\left[\sup _{s \leqslant T}\left|X_{s}^{n+1}-X_{s}^{n}\right|^{2}\right] \leqslant \frac{\left(C_{\alpha, T}\right)^{n}}{n !},
$$

which yields

$$
P\left(\sup _{s \leqslant T}\left|X_{s}^{n+1}-X_{s}^{n}\right|>\frac{1}{4^{n}}\right) \leqslant \frac{\left(4 C_{\alpha, T}\right)^{n}}{n !} .
$$

Using the Borel-Cantelli lemma, it follows that $X_{t}^{n}$ converges uniformly to a continuous, adapted process $X$ on $[0, T]$ almost surely. Again by the Borel-Cantelli argument, it is also seen that $M_{n}(t):=\int_{0}^{t} \sigma\left(X_{s}^{n}\right) d B_{s}$ converges uniformly on [0,T] to $M(t)=\int_{0}^{t} \sigma\left(X_{s}\right) d B_{s}$ almost surely. Thus, by (57), we see that $L_{t}^{n}$ converges uniformly to a continuous non-decreasing process $L$ on $[0, T]$ almost surely. Letting $n \rightarrow \infty$ in (57) gives

$$
X_{t}=\int_{0}^{t} \sigma\left(X_{s}\right) d B_{s}+\alpha \max _{0 \leqslant s \leqslant t} X_{s}+L_{t} .
$$

To show that $\left(X_{t}, L_{t}\right)$ is a solution to $(45)$, we need to prove

$$
\int_{0}^{t} \chi_{\left\{X_{s}=0\right\}} d L_{s}=L_{t} .
$$


This will follow if we can show that for any $f \in C_{0}(0, \infty)$

$$
\int_{0}^{t} f\left(X_{s}\right) d L_{s}=0
$$

Indeed,

$$
\int_{0}^{t} f\left(X_{s}\right) d L_{s}=\lim _{n \rightarrow \infty} \int_{0}^{t} f\left(X_{s}^{n}\right) d L_{s}^{n}=0 .
$$

Next we show the uniqueness. Let $\left(X_{t}^{1}, L_{t}^{1}\right),\left(X_{t}^{2}, L_{t}^{2}\right)$ be two solutions to Eq. (45). Using similar arguments as above, it can be shown that

$$
E\left[\sup _{s \leqslant t}\left|X_{s}^{1}-X_{s}^{2}\right|^{2}\right] \leqslant C_{\alpha} E\left[\int_{0}^{t}\left(X_{s}^{1}-X_{s}^{2}\right)^{2} d u\right] .
$$

By Gronwall's inequality, it follows that $X^{1}=X^{2}$, and hence $L^{1}=L^{2}$.

Remark 4.4. Under the conditions in Theorem 4.3 the existence can also be obtained from Theorem 4.2 by letting $x \rightarrow 0$ and showing the corresponding solutions form a Cauchy sequence in the $L^{2}(\Omega)$-space.

Now, consider the stochastic differential equation:

$$
X_{t}=x+\int_{0}^{t} \sigma\left(X_{s}\right) \circ d B_{s}+\alpha \max _{0 \leqslant s \leqslant t} X_{s}+L_{t},
$$

where $\circ$ means the Stratonovich integration. A solution to this equation is defined as in Definition 4.1 with Ito integration replaced by the Stratonovich integration.

Theorem 4.5. Assume that there exists a constant $c>0$ such that $\sigma(x) \geqslant c$, and $\frac{d \sigma(x)}{d x}$ is continuous. Then for any $0 \leqslant \alpha<1$ and $x \geqslant 0$ there exists a unique solution $\left(X_{t}, L_{t}, t \geqslant 0\right)$ to Eq. (67).

Proof. We prove the theorem for $x=0$. Other cases are similar. Introduce two functions $F_{1}(x), F_{2}(x)$ on $R$ defined by

$$
F_{1}(x)=\int_{0}^{x} \frac{1}{\sigma(u)} d u, \quad F_{2}(x)=\int_{0}^{x} \sigma\left(F_{2}(u)\right) d u .
$$

Note that $F_{1}, F_{2} \in C^{2}(R), F_{1}=F_{2}^{-1}$, and

$$
\begin{array}{ll}
F_{1}(x)=0 \Leftrightarrow x=0, & F_{1}(x) \geqslant 0 \Leftrightarrow x \geqslant 0, \\
F_{2}(x)=0 \Leftrightarrow x=0, & F_{2}(x) \geqslant 0 \Leftrightarrow x \geqslant 0 .
\end{array}
$$

It follows from Section 3 (also [3]) that there is a unique solution, denoted by $\left(X_{t}^{0}, L_{t}^{0}\right)$, to the equation

$$
X_{t}^{0}=B_{t}+\alpha \max _{0 \leqslant s \leqslant t} X_{s}^{0}+L_{t}^{0}
$$


Define $X_{t}=F_{2}\left(X_{t}^{0}\right)$ and $L_{t}=\int_{0}^{t} \sigma\left(X_{s}\right) d L_{s}^{0}$. Put $m_{t}^{0}=\max _{0 \leqslant s \leqslant t} X_{s}^{0}$. By Itô's formula, we have that

$$
X_{t}=\int_{0}^{t} F_{2}^{\prime}\left(X_{s}^{0}\right) \circ d X_{s}^{0}=\int_{0}^{t} \sigma\left(X_{s}\right) \circ d B_{s}+\alpha \int_{0}^{t} \sigma\left(X_{s}\right) d m_{s}^{0}+L_{t} .
$$

Since $F_{2}$ is a monotone increasing function, it follows that

$$
\max _{0 \leqslant s \leqslant t} X_{s}=\max _{0 \leqslant s \leqslant t} F_{2}\left(X_{s}^{0}\right)=F_{2}\left(m_{t}^{0}\right) .
$$

On the other hand,

$$
\begin{aligned}
F_{2}\left(m_{t}^{0}\right) & =\int_{0}^{t} F_{2}^{\prime}\left(m_{s}^{0}\right) d m_{s}^{0}=\int_{0}^{t} \sigma\left(F_{2}\left(m_{s}^{0}\right)\right) d m_{s}^{0} \\
& =\int_{0}^{t} \sigma\left(F_{2}\left(X_{s}^{0}\right)\right) d m_{s}^{0}=\int_{0}^{t} \sigma\left(X_{s}\right) d m_{s}^{0},
\end{aligned}
$$

where we have used the fact that the measure $d m^{0}$ does not charge the set $\left\{s ; m_{s}^{0}>x_{s}^{0}\right\}$. Thus (71) becomes

$$
X_{t}=\int_{0}^{t} \sigma\left(X_{s}\right) \circ d B_{s}+\alpha \max _{0 \leqslant s \leqslant t} X_{s}+L_{t} .
$$

It is clear that $L_{t}, t \geqslant 0$ is a continuous, non-decreasing process. Moreover,

$$
\int_{0}^{t} \chi_{\{0\}}\left(X_{s}\right) d L_{s}=\int_{0}^{t} \chi_{\{0\}}\left(X_{s}^{0}\right) d L_{s}=\int_{0}^{t} \chi_{\{0\}}\left(X_{s}^{0}\right) \sigma\left(X_{s}\right) d L_{s}^{0}=L_{t} .
$$

This shows that $\left(X_{t}, L_{t}, t \geqslant 0\right)$ is a solution to Eq. (67).

On the other hand, similar arguments also show that for any solution $\left(X_{t}, L_{t}, t \geqslant 0\right)$ to Eq. $(67),\left(X_{t}^{0}=\right.$ $\left.F_{1}\left(X_{t}\right), L_{t}^{0}=\int_{0}^{t} \frac{1}{\sigma\left(X_{s}\right)} d L_{s}\right)$ is a solution to Eq. (70). Thus, the uniqueness of the solution to Eq. (67) is a consequence of the uniqueness of the solution to Eq. (70). The proof is complete.

\section{Acknowledgements}

We are grateful to the anonymous referee for very careful reading and useful suggestions. This work is partially supported by an EPSRC grant (no. GR/R91144).

\section{References}

[1] Ph. Carmona, F. Petit, M. Yor, Some extentions of the arcsine law as (partial) consequences of the scaling property of Brownian motion, Probab. Theory Related Fields 100 (1994) 1-29.

[2] Ph. Carmona, F. Petit, M. Yor, Beta variables as times spent in $[0, \infty)$ by certain perturbed Brownian motions, J. London Math. Soc. 58 (1998) 239-256.

[3] L. Chaumont, R.A. Doney, Pathwise uniqueness for perturbed versions of Brownian motion and reflected Brownian motion, Probab. Theory Related Fields 113 (1999) 519-534.

[4] L. Chaumont, R.A. Doney, Some calculations for doubly perturbed Brownian motion, Stochastic Process. Appl. 85 (1) (2000) 61-74. 
[5] B. Davis, Weak limits of perturbed random walks and the equation $Y_{t}=B_{t}+\alpha \sup _{s \leqslant t} Y_{s}+\beta \inf _{s \leqslant t} Y_{s}$, Ann. Probab. 24 (1996) 20072023.

[6] B. Davis, Brownian motion and random walk perturbed at extrema, Probab. Theory Related Fields 113 (1999) 501-518.

[7] R.A. Doney, Some calculations for perturbed Brownian motion, in: Seminaire de Probabilites XXXII, in: Lecture Notes in Mathematics, 1998, pp. 231-236.

[8] N. Ikeda, S. Watanabe, Stochastic Differential Equations and Diffusion Processes, second ed., North-Holland/Kodansha, 1989.

[9] J.F. Le Gall, M. Yor, Excursions browniennes et carrés de processus de Bessel, C. R. Acad. Sci. Paris Sér. I 303 (1986) 73-76.

[10] J.F. Le Gall, M. Yor, Enlacements du mouvement brownien autour des courbes de l'espace, Trans. Amer. Math. Soc. 317 (1990) 687-7722.

[11] P.L. Lions, A.S. Sznitman, Stochastic differential equations with reflecting boundary conditions, Comm. Pure Appl. Math. 37 (1984) 511-537.

[12] M. Perman, W. Werner, Perturbed Brownian motions, Probab. Theory Related Fields 108 (1997) 357-383.

[13] D. Revuz, M. Yor, Continuous Martingales and Brownian Motion, second ed., Springer, Berlin, 1994.

[14] W. Werner, Some remarks on perturbed Brownian motion, in: Seminaire de Probabilites XXIX, in: Lecture Notes in Mathematics, vol. 1613,1995 , pp. 37-43.

[15] T.S. Zhang, On the strong solutions of one-dimensional stochastic differential equations with reflecting boundary, Stochastic Process. Appl. 50 (1994) 135-147. 